\begin{tabular}{|l|}
\hline Access this article online \\
\hline Quick Response Code: \\
\hline Website: \\
www.jponline.org \\
\hline DOI: \\
10.4103/JLP.JLP_27_19 \\
\hline
\end{tabular}

Department of Microbiology, Mahatma Gandhi Medical College and Research Institute,

Sri Balaji Vidyapeeth (Deemed University). Puducherry, India

Address for correspondence:

Dr. Arunava Kali,

Department of Microbiology, Mahatma Gandhi Medical College and Research Institute,

Sri Balaji Vidyapeeth (Deemed University),

Puducherry - 607 402, India.

E-mail: ak.arunava@ gmail.com

Submission: 17-02-2019 Accepted: 23-08-2019

\title{
Evaluation of in vitro susceptibility of fosfomycin among Enterobacteriaceae isolates from urine cultures: A study from Puducherry
}

\author{
Srirangaraj Sreenivasan, Arunava Kali, M. V. Pravin Charles, Seetha Kunigal
}

\begin{abstract}
:
INTRODUCTION: The increasing drug resistance among Gram-negative uropathogens and a lack of effective oral antibiotics have limited the therapeutic options available for urinary tract infections (UTIs). This shortage of newer antibiotics has paved the way for considering the use of older antibiotics such as fosfomycin. This study aims to evaluate the in vitro susceptibility of Enterobacteriaceae isolates to fosfomycin.
\end{abstract}

MATERIALS AND METHODS: In this descriptive study conducted over a period of 6 months, we processed 1500 urine samples. The Enterobacteriaceae isolates were subjected to in vitro susceptibility testing to fosfomycin, in addition to the regularly used urinary antibiotics, by Kirby-Bauer disc-diffusion method.

RESULTS: Of 1500 urine samples processed, 582 samples yielded the growth of pathogens. Enterobacteriaceae accounted for $392(67.3 \%)$ of the isolates. Among these isolates, lower rates of resistance were observed for imipenem $(4.1 \%)$ and fosfomycin $(13.3 \%)$. Relatively higher rates of resistance were observed for nitrofurantoin (35.5\%) and amikacin (30.9\%). Nalidixic acid, norfloxacin, gentamicin, cefotaxime, and cotrimoxazole showed a high resistance rate of $82.7 \%, 69.6 \%, 52.3 \%$, $69.1 \%$, and $71.4 \%$, respectively. All antibiotics, except fosfomycin, were in routine clinical use in our hospital. The low resistance (13.3\%) to fosfomycin is indicative of its utility as an excellent urinary antibiotic.

CONCLUSIONS: Uropathogenic Enterobacteriaceae isolates displayed excellent in vitro susceptibility to fosfomycin. These in vitro findings suggest the unexplored potential of fosfomycin as a superior therapeutic option for treating uncomplicated UTI.

Key words:

Enterobacteriaceae, fosfomycin, urinary tract infection

\section{Introduction}

丁ring rinary tract infection (UTI) is a common ailment diagnosed by clinicians in day-to-day practice in the community as well as in health-care setups. Although the risk factors and patient groups differ in community and hospitals, Gram-negative bacteria, especially Enterobacteriaceae, account for a

This is an open access journal, and articles are distributed under the terms of the Creative Commons Attribution-NonCommercial-ShareAlike 4.0 License, which allows others to remix, tweak, and build upon the work non-commercially, as long as appropriate credit is given and the new creations are licensed under the identical terms.

For reprints contact: reprints@medknow.com large majority of UTI episodes in both the settings. Cotrimoxazole has long been used for the treatment of uncomplicated UTI in the community. However, over decades of use, it has lost its therapeutic efficacy against Gram-negative uropathogens in most parts of the world. Simple antibiotics such as oral preparations of fluoroquinolones and beta-lactams are currently preferred. However, the rapid development of resistance against quinolones and beta-lactam agents among

How to cite this article: Sreenivasan S, Kali A, Pravin Charles MV, Kunigal S. Evaluation of in vitro susceptibility of fosfomycin among Enterobacteriaceae isolates from urine cultures: A study from Puducherry. J Lab Physicians 2019;11:249-52. 
Enterobacteriaceae species recently in most countries has caused great concern for physicians.

High rates of resistance to nalidixic acid, ciprofloxacin, and norfloxacin have been reported in various studies. ${ }^{[1-3]}$ Due to the widespread emergence of extended-spectrum beta-lactamases (ESBLs), AmpC beta-lactamases, and carbapenemases, beta-lactam antibiotics are no longer reliable for empirical therapy in the absence of antibiogram. Although injectable antibiotics such as aminoglycosides, carbapenems, ureidopenicillins, polymyxin B, colistin, and tigecycline may show superior in vitro activity against uropathogens resistant to first-line drugs, they are reserved mainly for intractable UTI in hospitalized patients. Fosfomycin is an old antibiotic molecule developed in 1969 in Spain. ${ }^{[4]}$ Due to the wide antimicrobial spectrum of activity against Gram-positive and Gram-negative uropathogens and convenient oral regimen, it has regained its value for the treatment of UTI. In this current study, we evaluated the in vitro antibiotic activity of fosfomycin against uropathogenic Enterobacteriaceae isolates in comparison to other antibiotics.

\section{Materials and Methods}

A prospective study was performed in our tertiary care hospital in Pondicherry. Urine samples were obtained from both ambulatory and catheterized patients with suspected UTI from various medical and surgical inpatient and outpatient departments in our hospital. Informed consent was obtained from patients before the sample collection. Urine samples were collected aseptically and were sent immediately to the laboratory for further processing.

The samples were processed by standard microbiological procedures. The urine samples were cultured using semi-quantitative method by inoculating plating media (Cystine-lactose-electrolyte-deficient agar) using a calibrated loop. Following inoculation into the plates, wet mount preparations of the samples were observed under a microscope for the presence of pus cells, red blood cells, and microorganisms. After 18-24 h incubation of the cultured plates, colony count was recorded to determine significant bacteriuria. Non-duplicate bacterial isolates with significant colony counts were included in our study and were identified up to species level by biochemical tests. Antibiotic susceptibility was tested by Kirby-Bauer disc-diffusion method against a panel of antibiotics using bacterial suspension matched to 0.5 McFarland's standard as inoculum on Muller Hinton agar (MHA). The antibiotics used against Enterobacteriaceae isolates were cotrimoxazole $(1.25 / 23.75 \mu \mathrm{g})$, nitrofurantoin $(300 \mu \mathrm{g})$, nalidixic acid $(30 \mu \mathrm{g})$, norfloxacin $(10 \mu \mathrm{g})$, cefotaxime $(30 \mu \mathrm{g})$, gentamicin $(10 \mu \mathrm{g})$, amikacin $(30 \mu \mathrm{g})$, fosfomycin $(200 \mu \mathrm{g})$, and imipenem $(10 \mu \mathrm{g})$. The zones of inhibition on MHA plates were interpreted according to the Clinical and Laboratory Standards Institute guidelines. For quality control, Escherichia coli (ATCC 25922) was used. Enterobacteriaceae isolates showing cefotaxime zone $\leq 27 \mathrm{~mm}$ were considered as ESBL producers and were confirmed by combined disc test using ceftazidime $(30 \mu \mathrm{g})$ and ceftazidime-clavulanic acid discs $(30 / 10 \mu \mathrm{g})$.

\section{Results}

A total of 1500 urine samples were received in the department of microbiology of our hospital, for bacterial culture and antibiotic susceptibility from suspected cases of UTI during the study period. Of 1500 urine samples processed, 582 samples yielded the growth of pathogens. Patients of UTI were mostly from urology, obstetrics, and medicine wards $(30.2 \%, 28 \%$, and $21.6 \%$, respectively). A large fraction of the patients were females $(n=353$, $61.7 \%$ ) and $47.6 \%$ of the patients belonged to the age group of $21-40$ years.

The pathogens were E. coli $(n=290,49.8 \%)$, Klebsiella pneumoniae $(n=68,11.7 \%)$, Citrobacter diversus $(n=8$, $1.4 \%)$, Citrobacter freundii $(n=15,2.6 \%)$, Enterobacter cloacae $(n=3,0.5 \%)$, Proteus mirabilis $(n=4,0.7 \%)$, Proteus vulgaris $(n=4,0.7 \%)$, Pseudomonas aeruginosa $(n=22,3.8 \%)$, Acinetobacter baumannii $(n=27,4.6 \%)$, non-fermenter Gram-negative bacilli ( $n=24,4.1 \%)$, Enterococcus spp. $(n=63,10.8 \%)$, Staphylococcus spp. $(n=28,4.8 \%)$, and Candida spp. $(n=26,4.5 \%)$. Enterobacteriaceae accounted for $67.3 \%(n=392)$ of the isolates. The microorganism-wise antibiotic resistance pattern is detailed in Table 1. EBSL-producing isolates $(271,69.1 \%)$ which included 215 E. coli and 36 K. pneumoniae were identified by ESBL combined disc test. Fosfomycin susceptibility among these ESBL-producing E. coli and K. pneumoniae was found in $93 \%$ and $55.6 \%$, respectively. A total of 269 (68.6\%) of our isolates showed resistance to three or more antibiotic classes and were considered as multidrug-resistant (MDR). Fosfomycin resistance among these MDR strains is described in Table 2.

\section{Discussion}

Urinary infections are one of the commonest primary diagnoses in health-care settings. The patients often present with vague complaints and asymptomatic bacteriuria requiring the laboratory confirmation for successful treatment. At present, the emergence of resistance to regularly used antibiotics has left limited therapeutic options for UTI. Hence, there is an increasing need to develop and introduce new antimicrobials for this purpose. However, only a few newer antibiotics are in the pipeline of development. In this scenario, 
Table 1: Resistance pattern of Enterobacteriaceae isolates

\begin{tabular}{|c|c|c|c|c|c|c|c|c|c|}
\hline & $\begin{array}{c}\text { Imipenem, } \\
n(\%)\end{array}$ & $\begin{array}{c}\text { Nitrofurantoin, } \\
n(\%)\end{array}$ & $\begin{array}{c}\text { Norfloxacin, } \\
n(\%)\end{array}$ & $\begin{array}{l}\text { Nalidixic } \\
\text { acid, } n(\%)\end{array}$ & $\begin{array}{c}\text { Gentamicin, } \\
n(\%)\end{array}$ & $\begin{array}{c}\text { Amikacin, } \\
n(\%)\end{array}$ & $\begin{array}{c}\text { Cefotaxime, } \\
n(\%)\end{array}$ & $\begin{array}{c}\text { Fosfomycin, } \\
n(\%)\end{array}$ & $\begin{array}{c}\text { Cotrimoxazole, } \\
n(\%)\end{array}$ \\
\hline C. diversus $(n=8)$ & $1(12.5)$ & $5(62.5)$ & $5(62.5)$ & $6(75)$ & $4(50)$ & $2(25)$ & $5(62.5)$ & $2(25)$ & $5(62.5)$ \\
\hline C. freundii $(n=15)$ & 2 (13.3) & $11(73.3)$ & $10(66.7)$ & 11 (73.3) & $9(60)$ & $4(26.7)$ & 8 (53.3) & $1(6.7)$ & $9(60)$ \\
\hline E. coli $(n=290)$ & $4(1.4)$ & $68(23.4)$ & $225(77.6)$ & $265(91.4)$ & $154(53.1)$ & $84(29)$ & $215(74.1)$ & $19(6.6)$ & $213(73.4)$ \\
\hline E. cloacae $(n=3)$ & 0 & $1(33.3)$ & 0 & 0 & 1 (33.3) & 1 (33.3) & 1 (33.3) & 0 & 1 (33.3) \\
\hline $\begin{array}{l}\text { K. pneumoniae } \\
(n=68)\end{array}$ & 7 (10.3) & $46(67.6)$ & $28(41.2)$ & $35(51.5)$ & $34(50)$ & 24 (35.3) & $36(52.9)$ & $28(41.2)$ & $44(64.7)$ \\
\hline P. mirabilis $(n=4)$ & $1(25)$ & $4(100)$ & $2(50)$ & $3(75)$ & $1(25)$ & $4(100)$ & $2(50)$ & $1(25)$ & $4(100)$ \\
\hline P. vulgaris $(n=4)$ & $1(25)$ & $4(100)$ & $3(75)$ & $4(100)$ & $2(50)$ & $2(50)$ & $4(100)$ & $1(25)$ & $4(100)$ \\
\hline Total $(n=392)$ & $16(4.1)$ & $139(35.5)$ & $273(69.6)$ & $324(82.7)$ & 205 (52.3) & $121(30.9)$ & $271(69.1)$ & $52(13.3)$ & $280(71.4)$ \\
\hline
\end{tabular}

C. diversus=Citrobacter diversus, $C$. freundii=Citrobacter freundii, E. coli=Escherichia coli, E. cloacae=Enterobacter cloacae, $K$. pneumonia=Klebsiella pneumonia, P. mirabilis=Proteus mirabilis, $P$. vulgaris=Proteus vulgaris

Table 2: Fosfomycin resistance among multidrug-resistant isolates

\begin{tabular}{lc}
\hline MDR isolates & $\begin{array}{c}\text { Number of MDR isolates showing } \\
\text { fosfomycin resistance, } n(\%)\end{array}$ \\
\hline C. diversus $(n=5)$ & $1(20)$ \\
C. freundii $(n=9)$ & 0 \\
E. coli $(n=206)$ & $11(5.3)$ \\
E. cloacae $(n=1)$ & 0 \\
K. pneumoniae $(n=41)$ & $15(36.6)$ \\
P. mirabilis $(n=3)$ & $1(33.3)$ \\
P. vulgaris $(n=4)$ & $1(25)$ \\
Total $(n=269)$ & $29(10.8)$ \\
\hline
\end{tabular}

MDR=Multidrug resistant, $C$. diversus=Citrobacter diversus, $C$.

freundii=Citrobacter freundii, E. coli=Escherichia coli, E. cloacae $=$ Enterobacter cloacae, K. pneumonia=Klebsiella pneumonia, P. mirabilis=Proteus mirabilis,

$P$. vulgaris=Proteus vulgaris

fosfomycin, an older antibiotic, has reclaimed its utility in the treatment of UTI. ${ }^{[5]}$ Fosfomycin is a phosphonic acid derivative isolated from Streptomyces species which acts by inhibiting the first committed step in cell wall synthesis. ${ }^{[6]}$ It has a broad spectrum of antimicrobial activity against both Gram-positive and Gram-negative bacteria. It is non-toxic, well-tolerated, and is available in oral as well as intravenous formulations. In addition, its commercially available oral formulation (3 g single dose) is most convenient for treating UTI in outpatient departments. ${ }^{[7]}$

In the present study, we compared in vitro activity of fosfomycin with other antibiotics commonly used for treating UTI to evaluate its utility among the 392 isolates; nalidixic acid $(82.7 \%)$, cotrimoxazole $(71.4 \%)$, norfloxacin $(69.6 \%)$, cefotaxime $(69.1 \%)$, and gentamicin $(52.3 \%)$ showed high resistance rates [Table 1]. Lower rates of resistance were observed for imipenem $(4.1 \%)$, nitrofurantoin (35.5\%), and amikacin (30.9\%). Similar high resistance rates to oral antibiotics have been reported in other studies. ${ }^{[1,7,8]}$ A study from North India by Patwardhan and Singh found lower in vitro activity of ampicillin, amoxyclav, cotrimoxazole, nitrofurantoin, and norfloxacin. ${ }^{[7]}$ In a recent study from South India, Sardar et al. found $84.8 \%, 83.6 \%$, and $79 \%$ resistance to amoxicillin-clavulanic acid, cefixime, and norfloxacin in
170 uropathogenic E. coli isolates, while imipenem and methenamine mandelate had $100 \%$ sensitivity. ${ }^{[1]}$

The prevalence of ESBL-producing isolates in UTI varies from $21.8 \%$ to $64.8 \%$ in various studies. ${ }^{[9-11]}$ In this study, $69.1 \%(n=271)$ of our isolates were found to produce ESBL enzymes. This regional variation may be due to the differences in antibiotic usage and infection control measures in hospitals of different areas. The notable finding in our study is the remarkable antibacterial activity of fosfomycin against ESBL-producing uropathogens and strains which were resistant to other commonly used antibiotics. Only 13.3\% of our isolates were resistant to it. Fosfomycin sensitivity was noted among 236 (87.1\%) of ESBL-producing isolates which included $93 \%$ of ESBL-positive E. coli strains and to a lesser extent ESBL-positive K. pneumoniae (55.6\%). Likewise, only $5.3 \%$ and $36.6 \%$ of MDR E. coli and K. pneumoniae strains, respectively, were fosfomycin resistant [Table 2]. Superior sensitivity of fosfomycin observed in our study is in accordance with the findings of several other recent studies. ${ }^{[1,7,9,12]}$ In a recent study from an apex tertiary care centers of India, Patwardhan and Singh compared susceptibility of uropathogens against oral antibiotics and fosfomycin. ${ }^{[7]}$ Among 2783 non-repeating Enterobacteriaceae urinary isolates, $2730(98.1 \%)$ from patients of all ages and both sexes were fosfomycin sensitive. High in vitro susceptibility to fosfomycin was found among 96.5\% of ESBL-producing and $91.9 \%$ of metallo- $\beta$-lactamase-producing isolates. It was also effective against MDR isolates and Gram-positive isolates such as methicillin-resistant Staphylococcus aureus (MRSA). There are studies which showed in vitro susceptibility of fosfomycin against MDR pathogens such as MRSA, vancomycin-resistant enterococci, ESBL, and AmpC producers..$^{[10,12-14]}$

The low resistance to fosfomycin observed in our study may be due to the fact that fosfomycin was not in routine clinical use in our hospital unlike other antibiotics. Withdrawal of an antibiotic from routine usage or a prolonged period of therapeutic inoperativeness is more 
likely to remove the selective pressure on the antibiotic. ${ }^{[15]}$ Although the risk of selection of fosfomycin-resistant mutants and the development of resistance during therapy has limited its clinical over the past several years, this old antibiotic has been found to regain its antibacterial activity against bacterial pathogens in recent years in several countries including India where it has not been marketed. ${ }^{[7]}$ Currently, it is recommended for the treatment of uncomplicated UTI in adults and as antibiotic prophylaxis in transurethral diagnostic and surgical procedures. ${ }^{[16]}$ Fosfomycin has emerged as a major breakthrough in the treatment of UTI due to increasing proportion of colistin-resistant K. pneumoniae and Proteae with intrinsic nonsusceptibility to colistin in recent years. ${ }^{[16]}$ Furthermore, it was also found to exert an inhibitory effect on biofilms of MDR uropathogens that essentially plays an important role in the pathogenesis of UTI. ${ }^{[14]}$

However, fosfomycin susceptibility also depends on the consumption of the antibiotic. A changing trend with the varied result has been reported overtime where its consumption rate is high. In a study conducted in Spain showed an increase in resistance among E. coli urinary isolates where the consumption rate was up to $50 \%{ }^{[15]}$ Recently, ESBL-producing Enterobacteriaceae isolates were reported to carry fosfomycin-resistance determinant resulting in a higher fosfomycin-resistance rate. Wachino et al. from Japan identified Fos $A 3$ and FosC2, two novel fosfomycin-resistance determinants among CTX-M-producing E. coli isolates. ${ }^{[17]}$ Being located on a plasmid, these resistance genes are transferable and have the potential to disseminate high-level resistance to this antibiotic among other Gram-negative bacterial species.

\section{Conclusions}

Fosfomycin was the only oral antibiotic which had substantial in vitro antimicrobial activity against Enterobacteriaceae isolates in our study. ESBL-producing and MDR isolates were mostly sensitive to fosfomycin. Therefore, it could be a promising alternative to currently available first-line antibiotics for the treatment of uncomplicated UTI, especially for a naive population where the consumption rate of fosfomycin is nil.

\section{Financial support and sponsorship}

Nil.

\section{Conflicts of interest}

There are no conflicts of interest.

\section{References}

1. Sardar A, Basireddy SR, Navaz A, Singh M, Kabra V. Comparative evaluation of fosfomycin activity with other antimicrobial agents against $E$. coli isolates from urinary tract infections. J Clin Diagn Res 2017;11:DC26-9.

2. Ou LB, Nadeau L. Fosfomycin susceptibility in multidrug-resistant Enterobacteriaceae species and vancomycin-resistant enterococci urinary isolates. Can J Hosp Pharm 2017;70:368-74.

3. Keepers TR, Gomez M, Celeri C, Krause KM, Biek D, Critchley I. Fosfomycin and comparator activity against select Enterobacteriaceae, Pseudomonas, and Enterococcus urinary tract infection isolates from the United States in 2012. Infect Dis Ther 2017;6:233-43.

4. Raz R. Fosfomycin: An old - New antibiotic. Clin Microbiol Infect 2012;18:4-7.

5. Brown EM, Nathwani D. Antibiotic cycling or rotation: A systematic review of the evidence of efficacy. J Antimicrob Chemother 2005;55:6-9.

6. Hendlin D, Stapley EO, Jackson M, Wallick H, Miller AK, Wolf FJ, et al. Phosphonomycin, a new antibiotic produced by strains of Streptomyces. Science 1969;166:122-3.

7. Patwardhan V, Singh S. Fosfomycin for the treatment of drug-resistant urinary tract infections: Potential of an old drug not explored fully. Int Urol Nephrol 2017;49:1637-43.

8. Sastry S, Clarke LG, Alrowais H, Querry AM, Shutt KA, Doi Y. Clinical appraisal of fosfomycin in the era of antimicrobial resistance. Antimicrob Agents Chemother 2015;59:7355-61.

9. Sabharwal ER, Sharma R. Fosfomycin: An alternative therapy for the treatment of UTI amidst escalating antimicrobial resistance. J Clin Diagn Res 2015;9:DC06-9.

10. Sultan A, Rizvi M, Khan F, Sami H, Shukla I, Khan HM. Increasing antimicrobial resistance among uropathogens: Is fosfomycin the answer? Urol Ann 2015;7:26-30.

11. Banerjee S, Sengupta M, Sarker TK. Fosfomycin susceptibility among multidrug-resistant, extended-spectrum beta-lactamase-producing, carbapenem-resistant uropathogens. Indian J Urol 2017;33:149-54.

12. Seo MR, Kim SJ, Kim Y, Kim J, Choi TY, Kang JO, et al. Susceptibility of Escherichia coli from community-acquired urinary tract infection to fosfomycin, nitrofurantoin, and temocillin in Korea. J Korean Med Sci 2014;29:1178-81.

13. Neuner EA, Sekeres J, Hall GS, van Duin D. Experience with fosfomycin for treatment of urinary tract infections due to multidrug-resistant organisms. Antimicrob Agents Chemother 2012;56:5744-8.

14. Gopichand P, Agarwal G, Natarajan M, Mandal J, Deepanjali S, Parameswaran S, et al. In vitro effect of fosfomycin on multi-drug resistant gram-negative bacteria causing urinary tract infections. Infect Drug Resist 2019;12:2005-13.

15. Karageorgopoulos DE, Wang R, Yu XH, Falagas ME. Fosfomycin: Evaluation of the published evidence on the emergence of antimicrobial resistance in gram-negative pathogens. J Antimicrob Chemother 2012;67:255-68.

16. Freitas DM, Moreira DM. Fosfomycin trometamol vs ciprofloxacin for antibiotic prophylaxis before transrectal ultrasonography-guided prostate biopsy: A meta-analysis of clinical studies. Arab J Urol 2019;17:114-9.

17. Wachino J, Yamane K, Suzuki S, Kimura K, Arakawa Y. Prevalence of fosfomycin resistance among CTX-M-producing Escherichia coli clinical isolates in Japan and identification of novel plasmid-mediated fosfomycin-modifying enzymes. Antimicrob Agents Chemother 2010;54:3061-4. 